\title{
BMJ Open Safety of thrombolysis in stroke mimics: an observational cohort study from an urban teaching hospital in Sweden
}

\author{
Nikolaos Kostulas, ${ }^{1}$ Martin Larsson, ${ }^{1}$ Tor-Bjorn Kall, ${ }^{1}$ Mia von Euler, ${ }^{1,2}$ \\ David Nathanson ${ }^{1}$
}

To cite: Kostulas N, Larsson M, Kall T-B, et al. Safety of thrombolysis in stroke mimics: an observational cohort study from an urban teaching hospital in Sweden. BMJ Open 2017;7:e016311. doi:10.1136/ bmjopen-2017-016311

- Prepublication history for this paper is available online. To view these files, please visit the journal online (http://dx.doi. org/10.1136/bmjopen-2017016311).

Received 20 February 2017 Revised 17 August 2017 Accepted 22 August 2017

\section{(a) CrossMark}

${ }^{1}$ Depatment of Clinical Science and Education, Karolinska Institutet, Stockholm, Sweden

${ }^{2}$ Karolinska Institutet Stroke Research Network, Stockholm, Sweden

Correspondence to Dr David Nathanson; david.nathanson@sll.se

\section{ABSTRACT}

Objectives Acute stroke management has changed dramatically over the recent years, where a timely assessment is driven by the expanding treatment options of acute ischaemic stroke. This increases the risk in treating non-stroke patients (stroke mimics) with a possibly hazardous intravenous thrombolysis treatment (IVT).

Setting Patients of the thrombolysis registry of Södersjukhuset $A B$, a secondary health centre in Stockholm, were retrospectively studied to determine complications and outcome after IVT in strokes and stroke mimics.

Participants Consecutively, 674 recruited patients from 1 January 2008 to 1 December 2013 were analysed regarding demographics and outcome at 3 months after onset of symptoms.

Results Ischaemic stroke was confirmed in 625 patients (93\%), and 48 patients (7\%) were stroke mimics. Patients with strokes were older than stroke mimics 72 (IQR: 64-81) vs 54 years (IQR 40-67), p<0.0001. Antihypertensive and antithrombotic treatment were more common in patients with stroke $(p<0.0001$ and $p=0.006$, respectively). National Institute of Health Stroke Scale did not differ at time of presentation. Excellent outcome defined as modified Rankin Scale score 0-1, at 3 months, was less common in stroke than in stroke mimics $(50 \%$ vs $87.5 \%, p<0.0001)$. No stroke mimic had a symptomatic intracerebral haemorrhage. Age of less than 40 years may be a predictor for a patient to be a stroke mimic (OR: 8.7, $95 \%$ Cl: 3.2 to $24.0, p<0.0001$ ).

Conclusions Stroke mimics receiving IVT had a more favourable outcome compared with patients with stroke, and showed no haemorrhagic complications. Age below 40 years may be a predictor for stroke mimics.

\section{INTRODUCTION}

The management of acute stroke has changed dramatically over the last years. The expansion of intravenous thrombolysis treatment (IVT) given at most hospitals receiving patients with stroke, as well as the continued drip and ship paradigm may increase the risk of erroneous assessment of the acute patient. ${ }^{12}$ The struggle to decrease door-to-needle (DTN) time might increase

\section{Main strengths and limitations}

This paper is comprised of consecutive data over a large period of time, thus increasing sample size providing higher internal validity.

- The work-up of patients with stroke was done according to stroke guidelines also including followup by an experienced stroke doctor representing the natural setting in larger hospitals.

- This is retrospective, single centre study with external validity limitations.

- The work-up at time did not include MRI in most patients, possibly explaining the relative low number of found stroke mimics.

the risk of treating non-stroke patients even more. IVT comes with the risk of symptomatic intracerebral haemorrhage $(\mathrm{SICH})$ that may differ between $2 \%$ and $9 \%$ depending on the definition used. ${ }^{3-5}$ It is well described that several disorders such as migraine, vertigo and seizures may appear with symptoms such as paresis, speech disturbance and visual loss and thereby mimic a stroke. ${ }^{67}$ The proportion of stroke mimics in thrombolysis registries vary from $1 \%$ to $16 \% .^{8-12}$ In a meta-analysis of nine prospective studies, stroke mimicking patients were found to have a lower risk for intracerebral haemorrhage when compared with patients with true acute ischaemic stroke (RR: $0.33,95 \%$ CI: 0.14 to 0.77$)^{13}$ A retrospective single cohort study indicated that treating stroke mimics with IVT is safe. ${ }^{14}$ Also, a multicentre cohort study showed only $1 \%$ of SICH in stroke mimics compared with $7.9 \%$ in ischaemic stroke. ${ }^{15}$ MRI evaluation of the patient with acute stroke may increase the chance of discriminating between a stroke mimic and an actual stroke. ${ }^{16}$ However, this is limited by the availability of MRI scans in acute stroke, and due to the time it takes to assess an patient with an MRI scan. Scoring systems have been suggested to be used to 
differentiate between a stroke mimic and a stroke, and have been used in telestroke networks. ${ }^{17}$

Here we retrospectively evaluated the outcome of IVT in a consecutive thrombolysis cohort of Södersjukhuset AB, in Stockholm, Sweden. Demographic and outcome variables were described in stroke and stroke mimics, and predictors of the latter were determined.

\section{MATERIALS AND METHODS}

Patients were consecutively recruited at the Södersjukhuset $\mathrm{AB}$, a large teaching hospital in an urban area of Stockholm. All patients with stroke are primarily seen by the internal medicine doctor on call and during office hours also by a neurologist, who makes the final decision whether IVT will be given or not. Outside office hours, a neurologist is available on call and supports the majority of all IVT cases. Since January 2008, all patients receiving IVT have been prospectively followed up at 3 months and registered in a local thrombolysis registry. All patients receiving thrombolysis from 1 January 2008 to 1 December 2013 were retrospectively evaluated using the electronic and locally available thrombolysis database. All patient records were re-evaluated 3 months after sensor date (by DN) with regard to diagnosis and outcome after thrombolysis. Patients were described with regard to demographic parameters and laboratory parameters at admission and at follow-up at 3 months after onset of symptoms. Of 699 patients consecutively recruited in the thrombolysis registry, between January 2008 and December 2013, 674 were included in the final analysis.

\section{Data collection and clinical variables}

The evaluation of all patients at admission included collection of demographic data, medical history, vascular risk factors, National Institute of Health Stroke Scale (NIHSS) score on admission, modified Rankin Scale score (mRS) before the ischaemic event and biochemical test results. SICH was used according to the criteria of the National Institute of Neurological Disorders and Stroke trial $\left(\mathrm{SICH}_{\text {NINDS }}\right.$ : any haemorrhage plus any neurological deterioration). ${ }^{3}$ Follow-up evaluation was performed at 3 months after IVT by a stroke nurse, and included clinical and functional evaluation by NIHSS, mRS, measurement of blood pressure (BP) and body temperature and laboratory tests. Information on date of death was available in electronic records for all deceased patients. Patients who had received IVT on more than one occasion $(n=15)$ were included, but only the first time they received IVT was used in the final analysis.

Stroke mimics were determined using a set of clinical factors during hospital stay and follow-up (by the responsible $\mathrm{MD}$ at discharge and by Dr David Nathanson at follow-up), and usually including repetitive CT imaging according to clinical routine imaging after thrombolysis.

\section{Stroke and stroke mimics and risk factors}

Ischaemic stroke and transitory ischaemic attacks (TIA) were classified according to ICD-10. Arterial hypertension (HT) was considered present when the patients were on antihypertensive treatment on admission, or when HT was diagnosed by repeated measurements of systemic $\mathrm{BP}>140 / 90 \mathrm{~mm} \mathrm{Hg}$ during hospital stay. Diabetes mellitus (DM) was considered present when patients had a known diagnosis, and/or were on antidiabetic treatment on admission. Hyperlipidaemia was defined by the presence of statin treatment on admission or fasting total cholesterol $\geq 5.2 \mathrm{mmol} / \mathrm{L}$ or LDL cholesterol $\geq 2.6 \mathrm{mmol} / \mathrm{L}$. Atrial fibrillation was considered present when mentioned in patients' past medical history or present at admission ECG.

\section{Data analysis}

Normal distribution of the variables was tested with Shapiro-Wilk's test. Medians and IQR were used to describe the characteristics of the study participants. Differences in continuous variables among groups were investigated by the Mann-Whitney test, and categorical variables were analysed using the $\chi^{2}$ or Fishers exact test where appropriate. Logistic regression was used to investigate the associations between potential risk factors and outcome in stroke mimic. A Hosmer-Lemeshov goodness-of-fit test was used to examine whether the final multivariable models adequately fitted the data. Multiple tests correction has not been performed due to the exploratory purpose of the study. $p$ Values $<0.05$ were considered statistically significant. Analyses were done with SPSS V.20.0 (SPSS).

\section{RESULTS}

\section{Stroke and stroke mimic cohort}

A total of 674 patients were included in the final analysis, and 25 patients were excluded due to previous treatment with IVT. Ischaemic stroke was confirmed in 625 patients (93\%) based on clinical and imaging data, and 48 patients $(7 \%)$ were classified as stroke mimics after reviewing available clinical and imaging data.

Demographics, risk factors and clinical investigation profiles of the stroke and stroke mimic groups are presented in table 1.

Patients with strokes were significantly older than those with stroke mimics (72 (IQR: 64-81) vs 54 years (IQR $40-67), \mathrm{p}<0.0001)$, and prior medication for HT $(57 \%$ vs $28 \%, \mathrm{p}<0.0001)$ and antithrombotic treatment $(48 \%$ vs $28 \%, \mathrm{p}=0.006$ ) were more common in patients with stroke. HT was more common in stroke compared with stroke mimics ( $49 \%$ vs $25 \%, \mathrm{p}=0.001)$ and serum creatinine was higher in stroke ( 83 vs $75 \mu \mathrm{mol} / \mathrm{L}, \mathrm{p}=0.001$ ), whereas DM, atrial fibrillation and hyperlipidaemia did not differ between groups. 
Table 1 Baseline characteristics of strokes and stroke mimics treated with intravenous thrombolysis treatment

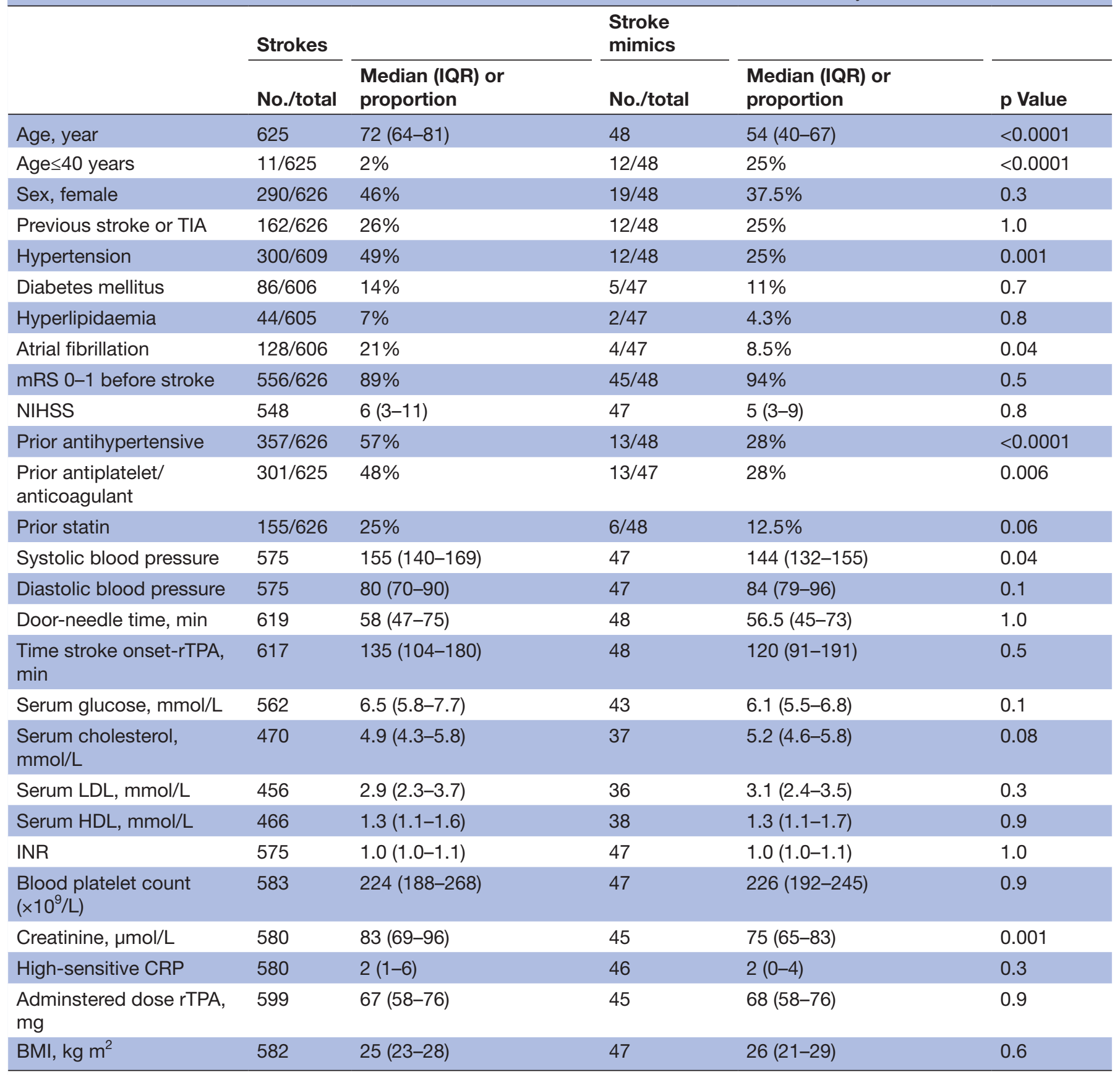

BMI, body mass index; mRS, modified Rankin Scale; NIHSS, National Institute of Health Stroke Scale; TIA, transitory ischaemic attacks.

\section{Stroke mimics}

Stroke mimics were determined after retrospectively reviewing medical records. Of the 674 patients, 48 patients $(7 \%)$ were diagnosed as a stroke mimic. Of these 48 patients, 12 (25\%) were determined functional with inorganic symptoms (functional mimics), 8 (17\%) were due to epileptic seizures, $10(21 \%)$ received symptom diagnoses (ie, a descriptive diagnosis without a determined aetiology) such as visual loss, eye muscle paresis, non-specific headache and paresis without cause, $4(8 \%)$ were diagnosed as alcohol intoxication, $3(6 \%)$ with migraine, $3(6 \%)$ with vertigo, 2 (4\%) with Bell's palsy, 2 (4\%) with hypotension,
$2(4 \%)$ with intracerebral tumour, $1(2 \%)$ with pain-related paresis and $1(2 \%)$ ischaemic heart disease. Of the 48 patients with stroke mimics, 13 of $270(4.8 \%)$ patients were treated during office time and 35 of $400(8.8 \%)$ patients were treated during on call time. However, on call time was not significantly associated with stroke mimicking neither in unadjusted (OR: $1.9,95 \%$ CI: 0.9 to 3.6 ) nor in multivariable models (OR: $1.8,95 \%$ CI: 0.9 to 3.7 , table 2 ).

\section{Clinical outcome and safety characteristics}

Baseline characteristics showed no differences in NIHSS between groups (table 1), but at 24 hours NIHSS was 
Table 2 Outcome and safety data after treatment with intravenous rTPA in strokes and stroke mimics

\begin{tabular}{|c|c|c|c|c|c|}
\hline & \multirow{2}{*}{$\begin{array}{l}\text { Strokes } \\
\text { No./total }\end{array}$} & \multirow[b]{2}{*}{$\begin{array}{l}\text { Median (IQR) or } \\
\text { proportion }\end{array}$} & \multirow{2}{*}{$\begin{array}{l}\begin{array}{l}\text { Stroke } \\
\text { mimics }\end{array} \\
\text { No./total }\end{array}$} & \multirow[b]{2}{*}{$\begin{array}{l}\text { Median (IQR) or } \\
\text { proportion }\end{array}$} & \multirow[b]{2}{*}{ p Value } \\
\hline & & & & & \\
\hline $\mathrm{SICH}_{\mathrm{NINDS}}$ & $11 / 542$ & $2 \%$ & $0 / 45$ & $0 \%$ & 1.0 \\
\hline mRS $0-1,3$ months & $258 / 513$ & $50 \%$ & $35 / 40$ & $87.5 \%$ & $<0.0001$ \\
\hline Mortality, 3 months & $69 / 595$ & $12 \%$ & $1 / 45$ & $2 \%$ & 0.048 \\
\hline Non-cerebral complications (all) & $27 / 562$ & $5 \%$ & $1 / 46$ & $2 \%$ & 0.7 \\
\hline Extracerebral haemorrhage & $16 / 562$ & $3 \%$ & $1 / 46$ & $2 \%$ & NA \\
\hline Hypotension (<90 mm Hg) & $2 / 562$ & $0.3 \%$ & $0 / 46$ & $0 \%$ & NA \\
\hline Nausea & $1 / 562$ & $0.2 \%$ & $0 / 50$ & $0 \%$ & NA \\
\hline Allergic reactions & $5 / 562$ & $0.8 \%$ & $0 / 50$ & $0 \%$ & NA \\
\hline Hospital stay, days & 599 & $5(3-8)$ & 47 & $4(2-7)$ & 0.3 \\
\hline NIHSS 24 hours after rTPA & 430 & $2(0-6)$ & 42 & $1(0-2)$ & 0.02 \\
\hline
\end{tabular}

mRS, modified Rankin Scale; NIHSS, National Institute of Health Stroke Scale; SICH, symptomatic intracerebral haemorrhage.

higher in stroke than stroke mimics (2 vs $1, \mathrm{p}=0.02$, table 2). There was no significant differences in SICH, 11 patients in the stroke group (2\%) suffered SICH and none of the stroke mimics. Extracerebral haemorrhage did not differ between groups either. Excellent outcome defined as mRS $0-1$ at 3 months was lower in stroke than in stroke mimics $(50 \%$ vs $87.5 \%, \mathrm{p}<0.0001)$. Mortality at 3 months was higher in stroke than stroke mimics $(12 \%$ vs $2 \%, \mathrm{p}<0.048)$

\section{Multivariate analysis of predictors of stroke mimics}

In order to determine prognostic variables to predict a stroke mimic, risk factors and lab parameters showing a significant difference between patients with stroke and stroke mimics (ie, age less than 40 years, HT and plasma creatinine) were included in a logistic multivariable model (table 3).

In this model, age below 40 years, adjusted for HT, atrial fibrillation and plasma creatinine, was significantly associated for being a stroke mimic $($ OR $8.7, \mathrm{p}<0.0001)$. HT and creatinine levels were also found to be independent predictors for being a stroke mimic, whereas

Table 3 Multivariable model with predictors of stroke mimics

\begin{tabular}{lll}
\hline Predictor & OR $(95 \%$ Cl) & p Value \\
\hline Age $<40$ & $8.7(3.2$ to 24.0$)$ & $<0.0001$ \\
\hline Hypertension & $0.5(0.2$ to 0.99$)$ & 0.047 \\
On call time & $1.8(0.9$ to 3.7$)$ & 0.09 \\
Atrial fibrillation & $0.5(0.2$ to 1.5$)$ & 0.2 \\
\hline Plasma creatinine $(\mu \mathrm{mol} / \mathrm{L})$ & $0.9(0.96$ to 0.99$)$ & 0.01 \\
\hline
\end{tabular}

atrial fibrillation was not a predictor of a stroke mimic (table 3).

\section{DISCUSSION}

This retrospective analysis of thrombolysed patients over 6 years, in accordance with previous literature, shows that stroke mimics are younger than stroke. ${ }^{15} 18$ DTN times did not differ between groups, possibly indicating that standardised protocols were in place. Haemorrhagic complications after IVT did not differ in groups, which has been shown before. ${ }^{319} 20$ Outcome measures such as NIHSS at 24 hours and mRS $0-1$ at 3 months indicated a worse functional status in the stroke group and also showed, as expected, higher mortality among strokes than stroke mimics. One fourth of the mimics were classified as functional mimics which is in line with other studies. ${ }^{21}$ Using multivariate analysis, predictors of stroke mimics were assessed and age below 40 was found to be the strongest predictor for a patient to be a stroke mimic (OR 8.7, p<0.0001), but also HT and creatinine levels (table 3) could indicate a patient to be a stroke mimic. The TeleStroke Mimic Score (TM Score) can discriminate strokes versus stroke mimics and includes age as a continuous variable, comorbidities such as atrial fibrillation and HT and NIHSS $>14 .{ }^{17}$ However, the TM Score may not be applicable in our stroke cohort due to lower NIHSS and the fact that we do not operate primarily within a telestroke network. Another potential risk factor for being a stroke mimic that was evaluated was the timepoint when admitted to hospital. We found a non-significant relative risk for being a stroke mimic of 1.8 for patients admitted to hospital outside office hours. As the majority of the patients in the present cohort (59\%) were admitted outside office hours, it is not possible to infer that admission time is a true risk factor for stroke mimicking. Nevertheless, we cannot rule out that our 
sample size is too small for answering this question. As there were no neurologists present at hospital outside office hours, it seems prudent to believe that a presence of specialists in neurology during on call time may reduce the risk for treating stroke mimicking patients with IVT.

The mean NIHSS for thrombolysed patients with stroke reported in the Swedish national quality register Riksstroke is $8 .^{22}$ Although our numbers of stroke mimics were fairly low in comparison with current literature indicating that as high as $20 \%$ of all patients presenting as suspected strokes are mimics, further radiological work-up with MRI could arguably exclude a stroke mimic, but also indicate a stroke when considered a mimic. MRI has a higher specificity (92\%) for arterial ischaemic stroke. A diffusion-weighted imaging MRI protocol has been shown to discriminated stroke mimics from arterial strokes. ${ }^{23-25}$ At our hospital the facilities did not at the time of the study allow rapid examination with MRI. The decrease in DTN times in recent years may also lead to higher frequencies of stroke mimics receiving thrombolysis, as could be shown in a single centre study that found an association between decreasing DTN times and increased frequencies of stroke mimics receiving IVT. ${ }^{26}$ This may warrant a better work-up of stroke presenting patients including the use of MRI. Moreover, there is a need for future studies with greater sample sizes for evaluating risk scores to discriminate patients with real stroke from those with stroke mimics. As previously described and showed also in our study, age is a potential variable to include in such scores.

\section{Limitations}

The first limitation is the retrospective character of our study. As a single centre study, the external validity may be limited. Although the internal validity is high as all follow-up and data collection were carried out by two trained nurses and only one doctor reevaluated the medical records. To enhance the generalisability, we extended the study period and consequently the sample size. The work-up of the patients not undergoing an MRI increases the chance of missing out on stroke mimics, which can also explain the relatively low number of stroke mimics although other single cohort studies have shown similar or lower levels. However, at this time and even today most acute stroke work-up includes CT, and stroke also remains a clinical diagnosis. Migraine aura might be visualised as a perfusion deficit on MRI and even though it has been reported to often involve several vascular territories it may still be mistaken as a stroke, even with the use of MRI. ${ }^{27}{ }^{28}$ At our centre, the low number of stroke mimics may also be due to assessment by an experienced stroke neurologist at daytime. The stroke mimics showed a multitude of different diagnoses and from a larger sample one might be able to draw conclusions on more common stroke mimic diagnoses.

The finding of an overrepresentation of young patients (ie, below 40 years of age) could reflect a higher likelihood to thrombolyse younger patients presenting with symptoms indicating a stroke. As the data are based on patients actually thrombolysed, the overrepresentation could be due to a higher willingness to thrombolyse rather than not thrombolyse in this age group if in doubt of the true diagnosis. Also, the likelihood of contraindication is lower in younger patients with less comorbidity. Retrospective studies from Europe and USA have found advanced age together with stroke severity to be the most common causes not to thrombolyse 2930

In conclusion, our retrospective cohort described relatively low numbers of stroke mimics, where low age may independently predict a patient to be a stroke mimic. Intravenous thrombolysis did not lead to significant complications in stroke mimics suggesting that the risk for IVT-associated complications in this group is low.

Correction notice This article has been corrected since it first published. The corresponding author has been changed to David Nathanson.

Acknowledgements The authors thank Natalia Trezie and Linda Ekström, research nurses at the Department of Internal Medicine Södersjukhuset AB, who meticulously recruited all patients and performed all data collection. They thank Lina Benson (Karolinska Institutet, Department of Clinical Science and Education, Södersjukhuset, Stockholm) for excellent statistical advice.

Contributors Guarantor of overall study integrity: NK and DN. Study concept and design: DN and NK. Data collection and interpretation: DN, NK, ML, T-BK and ME. Statistical analysis: DN, NK and ML. Manuscript preparation: NK, DN, ME, ML and T-BK. Final approval of manuscript: NK, DN, ME, ML (T-BK deceased).

Funding This study was supported by funds from Karolinska Institutet and the Fighting StrokeProject.

Competing interests None declared.

Patient consent Detail has been removed from this case description/these case descriptions to ensure anonymity. The editors and reviewers have seen the detailed information available and are satisfied that the information backs up the case the authors are making.

Ethics approval The study was approved by the regional ethical review board of Stockholm, EPN: 2012/626-31/4.

Provenance and peer review Not commissioned; externally peer reviewed.

Data sharing statement All authors agree on sharing data, none has been or is considered for publication elsewhere. Data available are that registered in the thrombolysis Registry of Södersjukhuset.

Open Access This is an Open Access article distributed in accordance with the Creative Commons Attribution Non Commercial (CC BY-NC 4.0) license, which permits others to distribute, remix, adapt, build upon this work non-commercially, and license their derivative works on different terms, provided the original work is properly cited and the use is non-commercial. See: http://creativecommons.org/ licenses/by-nc/4.0/

(c) Article author(s) (or their employer(s) unless otherwise stated in the text of the article) 2017. All rights reserved. No commercial use is permitted unless otherwise expressly granted.

\section{REFERENCES}

1. Hacke W, Kaste M, Bluhmki E, et al. Thrombolysis with alteplase 3 to 4.5 hours after acute ischemic stroke. N Engl J Med 2008;359:1317-29.

2. Martin-Schild S, Morales MM, Khaja AM, et al. Is the drip-and-ship approach to delivering thrombolysis for acute ischemic stroke safe? J Emerg Med 2011;41:135-41.

3. The National Institute of Neurological Disorders and Stroke rt-PA Stroke Study Group. Tissue plasminogen activator for acute ischemic stroke. N Engl J Med 1995;333:581-1587.

4. Sandercock P, Wardlaw JM, Lindley RI, et al. The benefits and harms of intravenous thrombolysis with recombinant tissue plasminogen activator within $6 \mathrm{~h}$ of acute ischaemic stroke (the third 
international stroke trial [IST-3]): a randomised controlled trial. Lancet 2012;379:2352-63.

5. Hacke W, Kaste M, Fieschi C, et al. Randomised double-blind placebo-controlled trial of thrombolytic therapy with intravenous alteplase in acute ischaemic stroke (ECASS II). Second European-Australasian Acute Stroke Study Investigators. Lancet 1998;352:1245-51.

6. Kidwell CS, Starkman S, Eckstein M, et al. Identifying stroke in the field. Prospective validation of the Los Angeles prehospital stroke screen (LAPSS). Stroke 2000;31:71-6.

7. Yew KS, Cheng EM. Diagnosis of acute stroke. Am Fam Physician 2015;91:528-36.

8. Artto V, Putaala J, Strbian D, et al. Stroke mimics and intravenous thrombolysis. Ann Emerg Med 2012;59:27-32.

9. Tsivgoulis G, Alexandrov AV, Chang J, et al. Safety and outcomes of intravenous thrombolysis in stroke mimics: a 6-year, singlecare center study and a pooled analysis of reported series. Stroke $2011 ; 42: 1771-4$

10. Uchino K, Massaro L, Hammer MD. Transient ischemic attack after tissue plasminogen activator: aborted stroke or unnecessary stroke therapy? Cerebrovasc Dis 2010;29:57-61.

11. Winkler DT, Fluri F, Fuhr P, et al. Thrombolysis in stroke mimics: frequency, clinical characteristics, and outcome. Stroke 2009;40:1522-5.

12. Chang J, Teleb M, Yang JP, et al. A model to prevent fibrinolysis in patients with stroke mimics. J Stroke Cerebrovasc Dis 2012;21:839-43.

13. Tsivgoulis G, Zand R, Katsanos AH, et al. Safety of intravenous thrombolysis in stroke mimics: prospective 5-year study and comprehensive meta-analysis. Stroke 2015;46:1281-7.

14. Sivakumaran P, Gill D, Mahir G, et al. A Retrospective Cohort Study on the use of intravenous thrombolysis in stroke mimics. J Stroke Cerebrovasc Dis 2016;25:1057-61.

15. Zinkstok SM, Engelter ST, Gensicke H, et al. Safety of thrombolysis in stroke mimics: results from a multicenter cohort study. Stroke 2013;44:1080-4.

16. Albers GW. Expanding the window for thrombolytic therapy in acute stroke. The potential role of acute MRI for patient selection. Stroke 1999;30:2230-7.

17. Ali SF, Viswanathan A, Singhal AB, et al. The TeleStroke mimic (TM)score: a prediction rule for identifying stroke mimics evaluated in a Telestroke Network. J Am Heart Assoc 2014;3:e000838.
18. Chen Y, Bogosavljevic V, Leys D, et al. Intravenous thrombolytic therapy in patients with stroke mimics: baseline characteristics and safety profile. Eur J Neurol 2011;18:1246-50.

19. Sandercock P, Wardlaw JM, Lindley RI, et al. The benefits and harms of intravenous thrombolysis with recombinant tissue plasminogen activator within $6 \mathrm{~h}$ of acute ischaemic stroke (the third international stroke trial [IST-3]): a randomised controlled trial. Lancet 2012;379:2352-63.

20. Hacke W, Kaste M, Fieschi C, et al. Randomised double-blind placebocontrolled trial of thrombolytic therapy with intravenous alteplase in acute ischaemic stroke (ECASS II). The Lancet 1998;352:1245-51.

21. Gargalas S, Weeks R, Khan-Bourne N, et al. Incidence and outcome of functional stroke mimics admitted to a hyperacute stroke unit. $J$ Neurol Neurosurg Psychiatry 2017;88.

22. Riksstroke annual report 2014 and 2015. Stroke och TIA, 2016. http://www.riksstroke.org/wp-content/uploads/2016/06/Riksstroke\% C3\%85rsrapport2015-PRELIMIN\%C3\%84R-WBB-\%C3\%A4ndrat6-juli.pdf

23. Chernyshev OY, Martin-Schild S, Albright KC, et al. Safety of tPA in stroke mimics and neuroimaging-negative cerebral ischemia. Neurology 2010;74:1340-5.

24. Brazzelli M, Sandercock PA, Chappell FM, et al. Magnetic resonance imaging versus computed tomography for detection of acute vascular lesions in patients presenting with stroke symptoms. Cochrane Database Syst Rev 2009;4:CD007424.

25. Eichel R, Hur TB, Gomori JM, et al. Use of DWI-only MR protocol for screening stroke mimics. J Neurol Sci 2013;328:37-40.

26. Liberman AL, Liotta EM, Caprio FZ, et al. Do efforts to decrease door-to-needle time risk increasing stroke mimic treatment rates? Neurol Clin Pract 2015;5:247-52.

27. Floery D, Vosko MR, Fellner FA, et al. Acute-onset migrainous aura mimicking acute stroke: MR perfusion imaging features. AJNR Am J Neuroradiol 2012;33:1546-52.

28. Förster $\mathrm{A}$, Wenz $\mathrm{H}$, Kerl HU, et al. Perfusion patterns in migraine with aura. Cephalalgia 2014;34:870-6.

29. Reiff T, Michel P. Reasons and evolution of non-thrombolysis in acute ischaemic stroke. Emerg Med J 2017;34:219-26.

30. Cappellari M, Bosco M, Forlivesi S, et al. Reasons for exclusion from intravenous thrombolysis in stroke patients admitted to the Stroke Unit. J Thromb Thrombolysis 2016;42:593-9. 